\title{
Imaging orbital ferromagnetism in a moiré Chern insulator
}

\author{
C. L. Tschirhart†, M. Serlin†, H. Polshyn, A. Shragaił, Z. Xia, J. Zhuł, Y. Zhang, K. Watanabe, T. Taniguchi, \\ M. E. Huber, A. F. Young*
}

'Department of Physics, University of California, Santa Barbara, CA 93106, USA. ${ }^{2}$ Research Center for Functional Materials, National Institute for Materials Science, 1-1 Namiki, Tsukuba 305-0044, Japan. IInternational Center for Materials Nanoarchitectonics, National Institute for Materials Science, 1-1 Namiki, Tsukuba 305-0044, Japan. ${ }^{4}$ Departments of Physics and Electrical Engineering, University of Colorado Denver, Denver, C0 80217, USA.

†These authors contributed equally to this work.

‡Present address: Department of Physics, Cornell University, Ithaca, NY 14853, USA.

${ }^{*}$ Corresponding author. Email: andrea@physics.ucsb.edu

Electrons in moiré flat band systems can spontaneously break time reversal symmetry, giving rise to a quantized anomalous Hall effect. Here we use a superconducting quantum interference device to image stray magnetic fields in twisted bilayer graphene aligned to hexagonal boron nitride. We find a magnetization of several Bohr magnetons per charge carrier, demonstrating that the magnetism is primarily orbital in nature. Our measurements reveal a large change in the magnetization as the chemical potential is swept across the quantum anomalous Hall gap consistent with the expected contribution of chiral edge states to the magnetization of an orbital Chern insulator. Mapping the spatial evolution of fielddriven magnetic reversal, we find a series of reproducible micron scale domains pinned to structural disorder.

In crystalline solids, orbital magnetization arises from the Berry curvature of the bands and intrinsic angular momentum of the Bloch electron wave packet (1). Although the orbital magnetization often contributes-at times substantially (2) - to the net magnetization of ferromagnets, all known ferromagnetism involves partial or full polarization of the electron spin. Theoretically, however, ferromagnetism can also arise through the spontaneous polarization of orbital magnetization without involvement of the electron spin. Recently, hysteretic transport consistent with ferromagnetic order has been observed in heterostructures composed of graphene and hexagonal boron nitride (3-7), neither of which are intrinsically magnetic materials. Notably, spin-orbit coupling is thought to be vanishingly small in these systems (8), effectively precluding a spin-based mechanism. These results have consequently been interpreted as evidence for purely orbital ferromagnetism (9-16).

To host purely orbital ferromagnetic order, a system must have a time reversal symmetric electronic degree of freedom separate from the electron spin as well as strong electronelectron interactions. Both are present in graphene heterostructures, where the valley degree of freedom provides degenerate electron species related by time reversal symmetry and a moiré superlattice can be used to engineer strong interactions. In these materials, a long wavelength moiré pattern, arising from interlayer coupling between mismatched lattices, modulates the underlying electronic structure and leads to the emergence of superlattice minibands within a reduced Brillouin zone. The small Brillouin zone means that low electron densities are sufficient to dope the 2D system to full filling or depletion of the superlattice bands, which can be achieved using experimentally realizable electric fields (17). For appropriately chosen constituent materials and interlayer rotational alignment, the lowest energy bands can have bandwidths considerably smaller than the native scale of electron-electron interactions, $E_{C} \approx e^{2} / \lambda_{M}$, where $\lambda_{M}$ is the moiré period and $e$ is the electron's charge. The dominance of interactions typically manifests experimentally through the appearance of "correlated insulators" at integer electron or hole filling of the moiré unit cell $(18,19)$, consistent with interaction-induced breaking of one or more of the spin, valley, or lattice symmetries. Orbital magnets are thought to constitute a subset of these states, in which exchange interactions favor a particular order that breaks time-reversal symmetry by causing the system to polarize into one or more valley projected bands. Remarkably, the large Berry curvature endows the valley projected bands with a finite Chern number $(20,21)$, so that valley polarization naturally leads to a quantized anomalous Hall effect at integer band filling. To date, quantum anomalous Hall effects have been observed at band fillings $v=1$ and $v=3$ in various heterostructures (46 ), where $v=A n$ corresponds to the number of electrons per unit cell area $A$ with $n$ the carrier density.

Although orbital magnetism is generally expected theoretically in twisted bilayer graphene (10-12), no direct experimental probes of magnetism have been reported because of the relative scarcity of magnetic samples, their small size, and the low expected magnetization density. The resulting 
magnetization density $m \lesssim 0.1 \mu_{\mathrm{B}} / \mathrm{nm}^{2}(22)$ (where $\mu_{\mathrm{B}} \approx 0.06$ $\mathrm{meV} / \mathrm{T}$ is the Bohr magneton) is consequently over three orders of magnitude smaller than in typical magnetic systems with several spins per subnanometer-sized crystal unit cell. The absence of magnetic studies leaves open both quantitative questions, such as the magnitude of the orbital magnetization, as well as qualitative ones regarding the nature of the magnetic phase transitions as a function of magnetic field and carrier density.

Here, we perform spatially resolved magnetometry to image the submicron magnetic structure of the same sample presented in (4) (see Fig. 1A), which consists of a twisted graphene bilayer aligned to one of the hexagonal boron nitride encapsulating layers. Figure 1B shows a schematic representation of our experimental setup. We use a superconducting quantum interference device (SQUID) fabricated on the tip of a quartz tube from cryogenically deposited indium (23) with a magnetic field sensitivity of approximately $15 \mathrm{nT} / \mathrm{Hz}^{1 / 2}$ at select out-of-plane magnetic fields of less than $50 \mathrm{mT}$ (see fig. S1). The SQUID is mounted to a quartz tuning fork (24, 25) (see fig. S2) and rastered in a 2D plane parallel to, and at a fixed height above, the tBLG heterostructure. A finite electrical excitation applied to the tuning fork generates a lateral oscillation of the tip along vector $\hat{a}$, and we measure the SQUID response at the tuning fork oscillation frequency, $B_{\mathrm{TF}} \approx \hat{a} \cdot \vec{\nabla}_{r} B_{z}$ (see fig. S3).

Figure $1, \mathrm{C}$ and $\mathrm{D}$, shows images of $B_{\mathrm{TF}}$ taken while the sample is doped to $n=2.36 \times 10^{12} \mathrm{~cm}^{-2}$, near the quantized Hall plateau corresponding to $v=3$. Here $n$ is the nominal density inferred from a parallel plate capacitor model, with the capacitance determined from the low-field Hall density (4). Images are acquired in the same background magnetic field $B=22 \mathrm{mT}$ but on opposite branches of the hysteresis loop shown in Fig. 1A. As discussed in (24) and fig. S7, the measured $B_{\mathrm{TF}}$ contains contributions from magnetic signals as well as other effects arising from electric fields or thermal gradients (24). To isolate the magnetic structure that gives rise to the observed hysteretic transport, we subtract data from Fig. 1C and Fig. 1D from each other. The result is shown in Fig. 1E, which depicts the gradient magnetometry signal associated with the fully polarized orbital ferromagnet. To reconstruct the static out-of-plane magnetic field, $B_{z}$, we integrate $B_{\mathrm{TF}}$ along $\hat{a}$ from the lower and left boundaries of the image (Fig. 1F). We infer the total magnetization density $m$ from the $B_{z}$ data using standard Fourier domain techniques (24) as shown in Fig. 1G. Figure 1, H and I, shows a comparison of $B_{\mathrm{TF}}$ and $m$ plotted along the contours indicated in Fig. 1, E and G. The shaded regions in Fig. II denote absolute error bounds from the dominant systematic uncertainty in $|\hat{a}|$.

Our measurements are taken close to $v=3$, equivalent to a single hole per unit cell relative to the nonmagnetic state at $v=4$ that corresponds to full filling of the lowest energy bands. We find that the magnetization density is considerably larger than $1 \mu_{\mathrm{B}}$ per unit cell area $A \approx 130 \mathrm{~nm}^{2}$, where we have taken $g=2$ as appropriate for graphene, in which spin orbit coupling is negligible. Without any assumptions about the nature of the broken symmetries, this state has a maximum spin magnetization of $1 \mu_{\mathrm{B}}$ per moiré unit cell. Our data reject this hypothesis, finding instead a maximum magnetization density of $m$ in the range $2-4 \mu_{\mathrm{B}}$ per moiré unit cell corresponding to an orbital magnetization of $1.8-3.6 \times 10^{-4} \mu_{\mathrm{B}} /$ carbon atom. We conclude that the magnetic moment associated with the QAH phase in tBLG is dominated by its orbital component.

In an intrinsic orbital magnet in which all moments arise from conduction electrons, the magnetization depends strongly on the density. Additional density dependence arises from the fact that contributions to the orbital magnetization from both wave-packet angular momentum and Berry curvature need not be uniformly distributed within the Brillouin zone (1). Transport observations of a quantum anomalous Hall effect measure only the total Berry curvature of a completely filled band. At partial band filling, however, extrinsic contributions from scattering complicate the relationship between transport and band properties. In contrast, measuring $m$ provides direct information about the density-dependent occupation of the Bloch states in momentum space. Figure $2 \mathrm{~A}$ shows repeated measurements of $B_{\mathrm{TF}}$ for a series of gate voltages in the vicinity of $v=3$. $B_{\mathrm{TF}}$ is measured along a contour that runs over a region of the device showing magnetic inhomogeneity even at the saturation magnetization (the black line cut in Fig. 1E; see also Fig. 1H). Assuming that the saturated magnetic state has density independent spatial structure [i.e., $m(n, r)=\mu_{\mathrm{B}} K(n) L(r)$, where $K$ and $L$ are respectively functions of density $\mathrm{n}$ and position $\mathrm{r}$ ], the amplitude of the position dependent modulation of $B_{\mathrm{TF}}$ functions as a proxy for $m$.

To compare magnetization at different $n$, we fit the data for $n=2.57 \times 10^{12} \mathrm{~cm}^{-2}$ to a 7 th order polynomial (see Fig. $2 \mathrm{~A}$ ); all other curves are then fit to the same polynomial with an overall scale factor which we denote $\tilde{B}_{\mathrm{TF}}$ and plot in Fig. $2 \mathrm{~B}$. Error bars reflect standard error of the mean of the residuals of these fits. $B_{\mathrm{TF}}$ is below our noise floor for $n \lesssim 2.25 \times 10^{12}$ $\mathrm{cm}^{-2}$. For $2.25 \times 10^{12} \mathrm{~cm}^{-2}<n<2.52 \times 10^{12} \mathrm{~cm}^{-2}-$ a density range that overlaps with the quantization of the Hall conductivity (4) $-B_{\mathrm{TF}}$ increases rapidly. For $n>2.52 \times 10^{12} \mathrm{~cm}^{-2}, B_{\mathrm{TF}}$ then slowly decreases as a function of $n$, dropping below the noise floor at $n \approx 2.9 \times 10^{12} \mathrm{~cm}^{-2}$, corresponding to a superlattice filling of $v \approx 3.7$ and approximately coinciding with the vanishing of ferromagnetic signatures in transport. Comparing the $n$-dependent $\tilde{B}_{\mathrm{TF}}$ with the residual Hall resistance shows that $\tilde{B}_{\mathrm{TF}}$ grows slowly as density is lowered toward $v$ $=3$, but then abruptly drops below the noise floor of our 
SQUID measurements within the range of $n$ associated with the quantized $R_{x y}$ plateau.

The dramatic change of the inferred $m$ within the quantum anomalous Hall plateau arises from the contribution of the chiral edge state to the total magnetization. Within an energy gap, changes in the chemical potential induce no additional charges in the sample bulk, and consequently no change in the bulk magnetization. In a Chern insulator, however, charges may accumulate on the sample boundary owing to the presence of chiral edge states. This is predicted to give rise to a chemical-potential dependent contribution to the magnetization within the quantized transport plateau (22). We interpret the sharp change we observe in magnetization within the transport plateau as evidence for this contribution to the magnetization by the topological edge states. Assuming $B_{\mathrm{TF}}$ to be a good proxy for $m$, our measurements imply a $\Delta m$ $\gtrsim 3 \mu_{\mathrm{B}} /$ u.c. across the quantum anomalous Hall gap. The magnitude of the theoretically expected jump in magnetization across a Chern insulator gap is $\Delta m=C E_{\text {gap }} / \Phi_{0}$, where $C$ is the Chern number, $E_{\text {gap }}$ is the topological band gap, and $\Phi_{0}=h / e$ is the flux quantum. Thermally activated transport measurements of the quantum anomalous Hall state at $v=3$ in this sample found $E_{\text {gap }}=2.5 \mathrm{meV}$ (4), corresponding to a $\Delta m$ of 1.4 $\mu_{\mathrm{B}}$ per unit cell, somewhat smaller than our local measurement and potentially implying that the transport measurements underestimate $E_{\text {gap }}$.

The orbital magnetization contributed by Bloch states within a valley-projected subband is momentum dependent. As a result, the total magnetization is expected to depend on electron density, although the precise trend is sensitive to the details of the many-particle ground state wave function. For $2.52 \times 10^{12} \mathrm{~cm}^{-2}<n<2.9 \times 10^{12} \mathrm{~cm}^{-2}$, the observed gradual decrease in $B_{\mathrm{TF}}$ is concomitant with the hysteresis in transport. In contrast, no $B_{\mathrm{TF}}$ signal is observed for $n<2.25 \times$ $10^{12} \mathrm{~cm}^{-2}$ despite hysteresis in transport persisting until $n=$ $2.05 \times 10^{12} \mathrm{~cm}^{-2}$. Under the assumption that $B_{\mathrm{TF}} \propto m$, absence of measured signal implies $|m|<.2 \mu_{\mathrm{B}}$ per moiré unit cell. Transport measurements in this density range show that the anomalous Hall effect changes sign at $n \approx 2.17 \times 10^{12} \mathrm{~cm}^{-2}$, accompanied by a divergence in the coercive field (fig. S8 and Fig. 2C). These phenomena, observed in a regime of undetectably small $m$, point to a density tuned transition in the valley occupation mediated by a sign change in the valley subband magnetization. In this picture, the sign change of the anomalous Hall effect arises directly from the opposite Berry curvatures of the contrasting valleys.

Although the coercive field behavior is difficult to model quantitatively, coercive fields in general result from competition between the energetic barrier to magnetic inversion and the coupling of magnetic order to the magnetic field: $B_{c} \sim$ $E / m$. For densities where $m$ approaches zero and $E$ does not, $B_{c}$ will to leading order be sensitive to changes in $1 / m$. The observation of a divergence in $B_{c}$ concomitant with a sign change in the anomalous Hall effect is consistent with a vanishing $m$ at this point. Our observation of an undetectably small magnetization in this regime strongly suggests these anomalies arise from a sign change in $m$ at partial band filling, rather than density-dependent extrinsic contributions to the anomalous Hall effect or domain pinning dynamics.

Across the range of densities at which magnetic hysteresis is observed, $B_{c}$ is not simply related to $m$, raising the question of the nature of magnetic pinning. Previous work on graphene-based Chern insulators has found Barkhausen noise jumps comparable to $h / e^{2}(3,4,6)$, suggesting a substructure of only a handful of ferromagnetic domains comparable in size to the distance between contacts. However, our magnetometry data show significant submicron scale inhomogeneity even at full magnetic saturation. This is similar to findings in transition metal doped topological insulators, where the magnetic structure is dominated by inhomogeneous distribution and clustering of the $\mathrm{Cr}$ or $\mathrm{V}$ dopants. In those systems, magnetic imaging shows superparamagnetic dynamics characterized by the reversal of weakly correlated point-like microscopic magnetic dipoles (26-28). Transport, meanwhile, does not typically show substantial Barkhausen noise (29), with the exception of one report where jumps were reported in a narrow range of temperatures (30).

To investigate the domain dynamics directly, we compare magnetic structure across different states stabilized in the midst of magnetic field driven reversal. Figure 3A shows a schematic depiction of our transport measurement, and Fig. 3B shows the resulting $R_{x y}$ data for both a major hysteresis loop spanning the two fully polarized states at $R_{x y}= \pm h / e^{2}$ (in purple) and a minor loop that terminates in a mixed polarization state at $R_{x y} \approx 0$ (in red). All three states represented by these hysteresis loops can be stabilized at $B=22 \mathrm{mT}$ for $T=$ $2.1 \mathrm{~K}$, where our nanoSQUID has excellent sensitivity, allowing a direct comparison of their respective magnetic structures (Fig. 3, C to E). Figure 3, F and G, shows images obtained by subtracting one of the images at full positive or negative polarization from the mixed state, as indicated in the lower left corners of the panels. Applying the same magnetic inversion algorithm used in Fig. 1 produces maps of $m$ corresponding to these differences (Fig. 3, H and I), allowing us to visualize the domain structure generating the intermediate plateau $R_{x y} \approx 0$ seen in the major hysteresis loop. The domains presented in Fig. 3, $\mathrm{H}$ and I, are difference images; the domain structures actually realized in experiment (Fig. 3, $\mathrm{C}$ to E) are illustrated schematically in Fig. 3, J to L. Evidently, the Hall resistance of the device in this state is dominated by the interplay of two large magnetic domains, each comprising about half of the active area.

Armed with knowledge of the domain structure, it is straightforward to understand the behavior of the measured 
transport in the mixed state imaged in Fig. 3D. In particular, the state corresponds to the presence of a single domain wall that crosses the device, separating both the current and the Hall voltage contacts (see Fig. 3A). In the limit in which the topological edge states at the boundaries of each magnetic domain are in equilibrium, there will be no drop in chemical potential across the domain wall, leading to $R_{x y}=0$. This is very close to the observed value of $R_{x y}=1.0 \mathrm{k} \Omega=0.039 h / e^{2}$. As shown in figs. S9 and S10, more subtle features of the transport curve can also be associated with the reversal of domains that do not bridge contacts (24).

In the absence of significant magnetic disorder ferromagnetic domain walls minimize surface tension. In two dimensions, domain walls are pinned geometrically in devices of finite size with convex internal geometry. As discussed in fig. S10 (24), we observe pinning of domain walls at positions that do not correspond to minimal length internal chords of our device geometry-suggesting that magnetic order couples to structural disorder directly. This is corroborated by the fact that the observed domain reversals associated with the Barkhausen jumps are consistent over repeated thermal cycles between cryogenic and room temperature.

Although crystalline defects on the atomic scale are unlikely in tBLG thanks to the high quality of the constituent graphene and hBN layers, the thermodynamic instability of magic angle twisted bilayer graphene makes it highly susceptible to inhomogeneity at scales larger than the moiré period, as shown in prior spatially resolved studies $(31,32)$. For example, the twist angle between the layers as well as their registry to the underlying $\mathrm{hBN}$ substrate may all vary spatially, providing potential pinning sites (33). Moiré disorder may thus be analogous to crystalline disorder in conventional ferromagnets, which gives rise to Barkhausen noise as it was originally described (34). A subtler issue raised by our data is the density dependence of magnetic pinning; as shown in Fig. $2, B_{c}$ does not simply track $1 / m$ across the entire density range, in particular failing to collapse with the rise in $m$ in the topological gap. This suggests nontrivial dependence of the pinning potential on the realized many body state. Understanding the pinning dynamics is critical for stabilizing magnetism in tBLG and the growing class of related orbital magnets, which includes both moiré systems (3-6) as well as more traditional crystalline systems such as rhombohedral graphite (35).

\section{REFERENCES AND NOTES}

1. D. Xiao, M.-C. Chang, Q. Niu, Berry phase effects on electronic properties. Rev. Mod. Phys. 82, 1959-2007 (2010). doi:10.1103/RevModPhys.82.1959

2. H. Adachi, H. Ino, A ferromagnet having no net magnetic moment. Nature 401, $148-$ 150 (1999). doi:10.1038/43634

3. A. L. Sharpe, E. J. Fox, A. W. Barnard, J. Finney, K. Watanabe, T. Taniguchi, M. A. Kastner, D. Goldhaber-Gordon, Emergent ferromagnetism near three-quarters filling in twisted bilayer graphene. Science 365, 605-608 (2019). doi:10.1126/science.aaw3780 Medline

4. M. Serlin, C. L. Tschirhart, H. Polshyn, Y. Zhang, J. Zhu, K. Watanabe, T. Taniguchi,
L. Balents, A. F. Young, Intrinsic quantized anomalous Hall effect in a moiré heterostructure. Science 367, 900-903 (2020). doi:10.1126/science.aay5533 Medline

5. G. Chen, A. L. Sharpe, E. J. Fox, Y.-H. Zhang, S. Wang, L. Jiang, B. Lyu, H. Li, K. Watanabe, T. Taniguchi, Z. Shi, T. Senthil, D. Goldhaber-Gordon, Y. Zhang, F. Wang, Tunable correlated Chern insulator and ferromagnetism in a moiré superlattice. Nature 579, 56-61 (2020). doi:10.1038/s41586-020-2049-7 Medline

6. H. Polshyn, J. Zhu, M. A. Kumar, Y. Zhang, F. Yang, C. L. Tschirhart, M. Serlin, K. Watanabe, T. Taniguchi, A. H. MacDonald, A. F. Young, Electrical switching of magnetic order in an orbital Chern insulator. Nature 588, 66-70 (2020). doj:10.1038/s41586-020-2963-8 Medline

7. S. Chen, M. He, Y.-H. Zhang, V. Hsieh, Z. Fei, K. Watanabe, T. Taniguchi, D. H. Cobden, X. Xu, C. R. Dean, M. Yankowitz, Electrically tunable correlated and topological states in twisted monolayer-bilayer graphene. Nat. Phys. 17, 374-380 (2020). doi:10.1038/s41567-020-01062-6

8. J. Sichau, M. Prada, T. Anlauf, T. J. Lyon, B. Bosnjak, L. Tiemann, R. H. Blick, Resonance microwave measurements of an intrinsic spin-orbit coupling gap in graphene: A possible indication of a topological state. Phys. Rev. Lett. 122, 046403 (2019). doi:10.1103/PhysRevLett.122.046403 Medline

9. M. Xie, A. H. MacDonald, Nature of the correlated insulator states in twisted bilayer graphene. Phys. Rev. Lett. 124, 097601 (2020). doi:10.1103/PhysRevLett.124.097601 Medline

10. N. Bultinck, S. Chatterjee, M. P. Zaletel, Mechanism for anomalous Hall ferromagnetism in twisted bilayer graphene. Phys. Rev. Lett. 124, 166601 (2020). doi:10.1103/PhysRevLett.124.166601 Medline

11. Y.-H. Zhang, D. Mao, T. Senthil, Twisted bilayer graphene aligned with hexagonal boron nitride: Anomalous Hall effect and a lattice model. Phys. Rev. Res. 1, 033126 (2019). doi:10.1103/PhysRevResearch.1.033126

12. J. Liu, X. Dai, Theories for the correlated insulating states and quantum anomalous Hall effect phenomena in twisted bilayer graphene. Phys. Rev. B 103, 035427 (2021). doi:10.1103/PhysRevB.103.035427

13. F. Wu, S. Das Sarma, Collective excitations of quantum anomalous Hall Ferromagnets in twisted bilayer graphene. Phys. Rev. Lett. 124, 046403 (2020). doi:10.1103/PhysRevLett.124.046403 Medline

14. S. Chatterjee, N. Bultinck, M. P. Zaletel, Symmetry breaking and skyrmionic transport in twisted bilayer graphene. Phys. Rev. B 101, 165141 (2020). doi:10.1103/PhysRevB.101.165141

15. C. Repellin, Z. Dong, Y.-H. Zhang, T. Senthil, Ferromagnetism in narrow bands of moiré superlattices. Phys. Rev. Lett. 124, 187601 (2020). doi:10.1103/PhysRevLett.124.187601 Medline

16. Y. Alavirad, J. Sau, Ferromagnetism and its stability from the one-magnon spectrum in twisted bilayer graphene. Phys. Rev. B 102, 235123 (2020). doi:10.1103/PhysRevB.102.235123

17. K. Kim, A. DaSilva, S. Huang, B. Fallahazad, S. Larentis, T. Taniguchi, K. Watanabe, B. J. LeRoy, A. H. MacDonald, E. Tutuc, Tunable moiré bands and strong correlations in small-twist-angle bilayer graphene. Proc. Natl. Acad. Sci. U.S.A. 114, 3364-3369 (2017). doi:10.1073/pnas.1620140114 Medline

18. Y. Cao, V. Fatemi, A. Demir, S. Fang, S. L. Tomarken, J. Y. Luo, J. D. SanchezYamagishi, K. Watanabe, T. Taniguchi, E. Kaxiras, R. C. Ashoori, P. Jarillo-Herrero, Correlated insulator behaviour at half-filling in magic-angle graphene superlattices. Nature 556, 80-84 (2018). doi:10.1038/nature26154 Medline

19. G. Chen, L. Jiang, S. Wu, B. Lyu, H. Li, B. L. Chittari, K. Watanabe, T. Taniguchi, Z. Shi, J. Jung, Y. Zhang, F. Wang, Evidence of a gate-tunable Mott insulator in a trilayer graphene moiré superlattice. Nat. Phys. 15, 237-241 (2019). doi:10.1038/s41567-018-0387-2

20. J. C. W. Song, P. Samutpraphoot, L. S. Levitov, Topological Bloch bands in graphene superlattices. Proc. Natl. Acad. Sci. U.S.A. 112, 10879-10883 (2015). doi:10.1073/pnas.1424760112 Medline

21. Y.-H. Zhang, D. Mao, Y. Cao, P. Jarillo-Herrero, T. Senthil, Nearly flat Chern bands in moiré superlattices. Phys. Rev. B 99, 075127 (2019). doi:10.1103/PhysRevB.99.075127

22. J. Zhu, J.-J. Su, A. H. MacDonald, Voltage-controlled magnetic reversal in orbital chern insulators. Phys. Rev. Lett. 125, 227702 (2020). doi:10.1103/PhysRevLett.125.227702 Medline 
23. Y. Anahory, H. R. Naren, E. O. Lachman, S. Buhbut Sinai, A. Uri, L. Embon, E. Yaakobi, Y. Myasoedov, M. E. Huber, R. Klajn, E. Zeldov, SQUID-on-tip with singleelectron spin sensitivity for high-field and ultra-low temperature nanomagnetic imaging. Nanoscale 12, 3174-3182 (2020). doi:10.1039/C9NR08578E Medline

24. See supplementary materials.

25. A. Uri, Y. Kim, K. Bagani, C. K. Lewandowski, S. Grover, N. Auerbach, E. O. Lachman, Y. Myasoedov, T. Taniguchi, K. Watanabe, J. Smet, E. Zeldov, Nanoscale imaging of equilibrium quantum Hall edge currents and of the magnetic monopole response in graphene. Nat. Phys. 16, 164-170 (2020). doi:10.1038/s41567-019$\underline{0713-3}$

26. E. O. Lachman, A. F. Young, A. Richardella, J. Cuppens, H. R. Naren, Y. Anahory, A. Y. Meltzer, A. Kandala, S. Kempinger, Y. Myasoedov, M. E. Huber, N. Samarth, E. Zeldov, Visualization of superparamagnetic dynamics in magnetic topological insulators. Sci. Adv. 1, e1500740 (2015). doi:10.1126/sciadv.1500740 Medline

27. E. O. Lachman, M. Mogi, J. Sarkar, A. Uri, K. Bagani, Y. Anahory, Y. Myasoedov, M. E. Huber, A. Tsukazaki, M. Kawasaki, Y. Tokura, E. Zeldov, Observation of superparamagnetism in coexistence with quantum anomalous Hall $\mathrm{C}= \pm 1$ and $\mathrm{C}=$ 0 Chern states. NPJ Quantum Mater. 2, 70 (2017). doi:10.1038/s41535-017-00721

28. K. Yasuda, M. Mogi, R. Yoshimi, A. Tsukazaki, K. S. Takahashi, M. Kawasaki, F. Kagawa, Y. Tokura, Quantized chiral edge conduction on domain walls of a magnetic topological insulator. Science 358, 1311-1314 (2017). doi:10.1126/science.aan5991 Medline

29. C.-Z. Chang, J. Zhang, X. Feng, J. Shen, Z. Zhang, M. Guo, K. Li, Y. Ou, P. Wei, L.-L. Wang, Z.-Q. Ji, Y. Feng, S. Ji, X. Chen, J. Jia, X. Dai, Z. Fang, S.-C. Zhang, K. He, Y. Wang, L. Lu, X.-C. Ma, Q.-K. Xue, Experimental observation of the quantum anomalous Hall effect in a magnetic topological insulator. Science 340,167-170 (2013). doi:10.1126/science.1234414 Medline

30. M. Liu, W. Wang, A. R. Richardella, A. Kandala, J. Li, A. Yazdani, N. Samarth, N. P. Ong, Large discrete jumps observed in the transition between Chern states in a ferromagnetic topological insulator. Sci. Adv. 2, e1600167 (2016). doi:10.1126/sciadv.1600167 Medline

31. A. Uri, S. Grover, Y. Cao, J. A. Crosse, K. Bagani, D. Rodan-Legrain, Y. Myasoedov, K. Watanabe, T. Taniguchi, P. Moon, M. Koshino, P. Jarillo-Herrero, E. Zeldov, Mapping the twist-angle disorder and Landau levels in magic-angle graphene. Nature 581, 47-52 (2020). doi:10.1038/s41586-020-2255-3 Medline

32. T. Benschop, T. A. de Jong, P. Stepanov, X. Lu, V. Stalman, S. J. van der Molen, D. K. Efetov, M. P. Allan, Measuring local moiré lattice heterogeneity of twisted bilayer graphene. Phys. Rev. Res. 3, 013153 (2021). doi:10.1103/PhysRevResearch.3.013153

33. Y. H. Kwan, G. Wagner, N. Chakraborty, S. H. Simon, S. A. Parameswaran, Domain wall competition in the Chern insulating regime of twisted bilayer graphene. arXiv:2007.07903 [cond-mat.str-el] (23 November 2020).

34. H. Barkhausen, Zwei mit Hilfe der neuen Verstrker entdeckte Erscheinungen. Phys. Ztschr. 20, 401 (1919)

35. Y. Shi, S. Xu, Y. Yang, S. Slizovskiy, S. V. Morozov, S.-K. Son, S. Ozdemir, C. Mullan, J. Barrier, J. Yin, A. I. Berdyugin, B. A. Piot, T. Taniguchi, K. Watanabe, V. I. Fal'ko, K. S. Novoselov, A. K. Geim, A. Mishchenko, Electronic phase separation in multilayer rhombohedral graphite. Nature 584, 210-214 (2020). doi:10.1038/s41586-020-2568-2 Medline

36. C. L. Tschirhart, M. Serlin, H. Polshyn, A. Shragai, Z. Xia, J. Zhu, Y. Zhang, K. Watanabe, T. Taniguchi, M. Huber, A. Young, Imaging orbital ferromagnetism in a moire Chern insulator, Dryad, Dataset (2021); https://doi.org/10.25349/D9JC8B.

37. A. Finkler, Y. Segev, Y. Myasoedov, M. L. Rappaport, L. Ne'eman, D. Vasyukov, E. Zeldov, M. E. Huber, J. Martin, A. Yacoby, Self-aligned nanoscale SQUID on a tip. Nano Lett. 10, 1046-1049 (2010). doi:10.1021/nl100009r Medline

38. D. Vasyukov, Y. Anahory, L. Embon, D. Halbertal, J. Cuppens, L. Neeman, A. Finkler, Y. Segev, Y. Myasoedov, M. L. Rappaport, M. E. Huber, E. Zeldov, A scanning superconducting quantum interference device with single electron spin sensitivity. Nat. Nanotechnol. 8, 639-644 (2013). doi:10.1038/nnano.2013.169 Medline

39. M. E. Huber, P. A. Neil, R. G. Benson, D. A. Burns, A. M. Corey, C. S. Flynn, Y. Kitaygorodskaya, O. Massihzadeh, J. M. Martinis, G. C. Hilton, DC SQUID series array amplifiers with $120 \mathrm{MHz}$ bandwidth. IEEE Trans. Appl. Supercond. 11, 1251-
1256 (2001). doi:10.1109/77.919577

40. A. Finkler, D. Vasyukov, Y. Segev, L. Ne'eman, E. O. Lachman, M. L. Rappaport, Y. Myasoedov, E. Zeldov, M. E. Huber, Scanning superconducting quantum interference device on a tip for magnetic imaging of nanoscale phenomena. Rev. Sci. Instrum. 83, 073702 (2012). doi:10.1063/1.4731656 Medline

41. D. Halbertal, M. Ben Shalom, A. Uri, K. Bagani, A. Y. Meltzer, I. Marcus, Y. Myasoedov, J. Birkbeck, L. S. Levitov, A. K. Geim, E. Zeldov, Imaging resonant dissipation from individual atomic defects in graphene. Science 358, 1303-1306 (2017). doi:10.1126/science.aan0877 Medline

42. E. Kleinbaum, G. A. Csáthy, Note: A transimpedance amplifier for remotely located quartz tuning forks. Rev. Sci. Instrum. 83, 126101 (2012). doi:10.1063/1.4769271 Medline

43. D. Sarid, Scanning Force Microscopy with Applications to Electric, Magnetic, and Atomic Forces (Oxford Univ. Press, 1991).

44. L. Thiel, Z. Wang, M. A. Tschudin, D. Rohner, I. Gutiérrez-Lezama, N. Ubrig, M. Gibertini, E. Giannini, A. F. Morpurgo, P. Maletinsky, Probing magnetism in 2D materials at the nanoscale with single-spin microscopy. Science 364, 973-976 (2019). doi:10.1126/science aav6926 Medline

45. B. J. Roth, N. G. Sepulveda, J. P. Wikswo Jr., Using a magnetometer to image a two-dimensional current distribution. J. Appl. Phys. 65, 361-372 (1989). doi:10.1063/1.342549

\section{ACKNOWLEDGMENTS}

The authors thank A. H. Macdonald, J. Zhu, M. Zaletel, and D. Xiao for discussions of the results and E. Lachman for their comments on the manuscript. Funding: The work was primarily funded by the Department of Energy under DE-SC0020043, with additional support for instrumentation development supported by the Army Research Office under Grant No. W911NF-16-1-0361. K.W. and T.T. acknowledge support from the Elemental Strategy Initiative conducted by the MEXT, Japan, Grant Number JPMXP0112101001, JSPS KAKENHI Grant Numbers JP2OH00354 and the CREST (JPMJCR15F3), JST. C.L.T. acknowledges support from the Hertz Foundation and from the National Science Foundation Graduate Research Fellowship Program under grant 1650114. This project is funded in part by the Gordon and Betty Moore Foundation's EPiQS Initiative, Grant GBMF9471 to A.F.Y. Author contributions: H.P. and Y.Z. fabricated the graphene heterostructure. C.L.T., M.S., and Z.X. fabricated the scanning probe tips. C.L.T., M.S., A.S., Z.X., J.Z., and A.F.Y. constructed the scanning probe microscope. M.S., A.S., and J.Z. wrote the microscope control software. M.E.H. provided the cryogenic amplifier. C.L.T., M.S., and A.F.Y. performed the measurements. C.L.T., M.S., Z.X., and A.F.Y. analyzed the data. T.T. and K.W. provided the hBN crystals. C.L.T., M.S., H.P., Z.X., and A.F.Y. wrote the manuscript. Competing interests: The authors declare no competing interests. Data and materials availability: All data shown in the main text and supplementary materials are available in the Dryad data repository (36)

\section{SUPPLEMENTARY MATERIALS}

science.sciencemag.org/cgi/content/full/science.abd3190/DC1

Materials and Methods

Supplementary Text

Figs. S1 to S11

References (37-45)

14 June 2020; accepted 13 May 2021

Published online 27 May 2021

10.1126/science.abd3190 

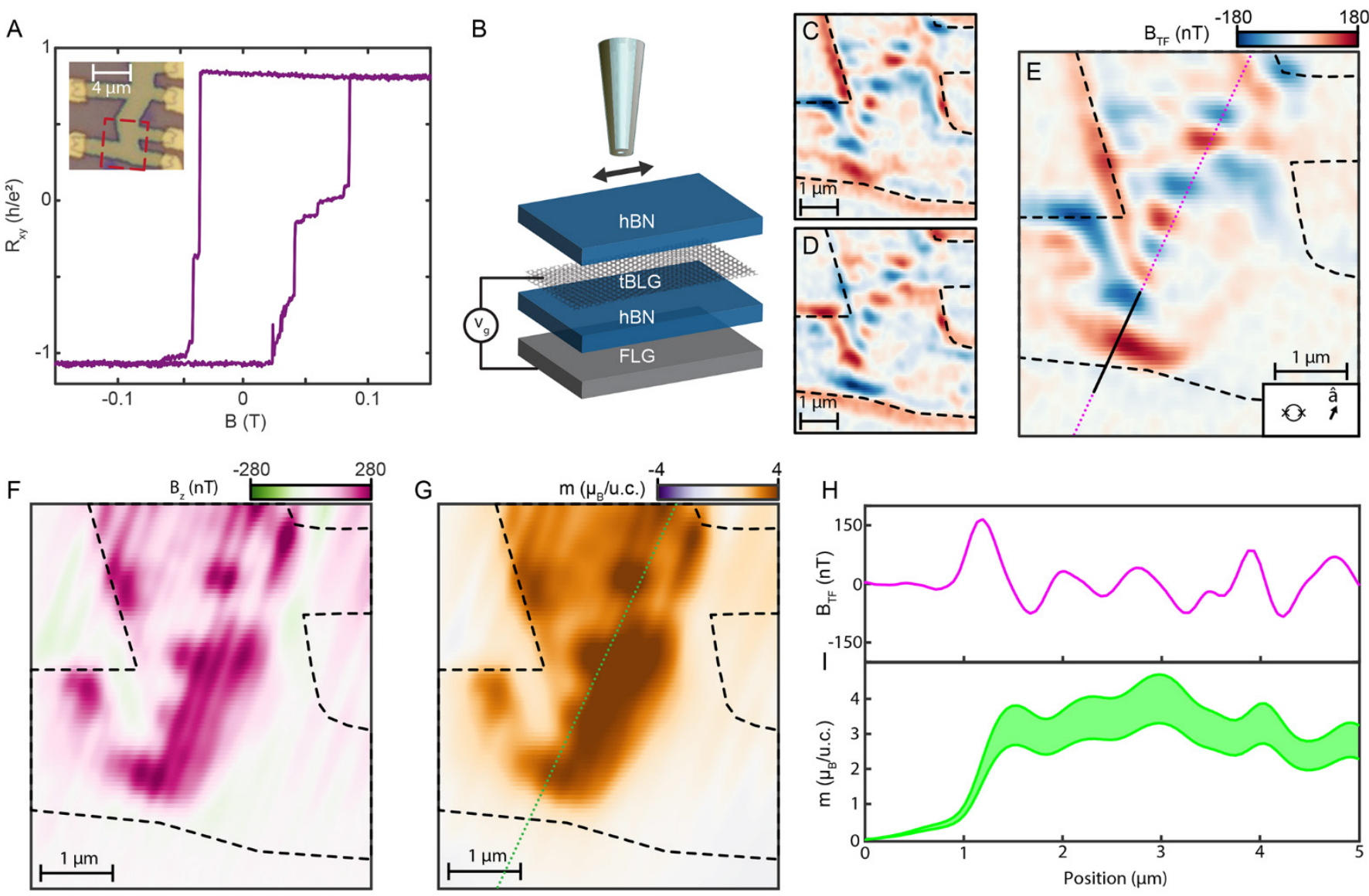

Fig. 1. Imaging orbital ferromagnetism. (A) Hall resistance of a twisted bilayer graphene device aligned to hexagonal boron nitride measured at $T=2.1 \mathrm{~K}$ and nominal electron density $n=2.36 \times 10^{12} \mathrm{~cm}^{-2}$. A thorough characterization of transport in this exact device is reported in (4). Inset: Optical micrograph of the device with the scan region marked in red. The scale bar is $4 \mu \mathrm{m}$. (B) Schematic illustration of the experimental setup. We raster a nanoscale indium SQUID with diameter $d=215 \mathrm{~nm}$ at a height of $h \approx 153 \mathrm{~nm}$ above the plane of the twisted bilayer graphene. The SQUID is coupled to a quartz tuning fork whose excitation causes the tip to oscillate mechanically at $f_{\mathrm{TF}} \approx 33 \mathrm{kHz}$ in the plane of the sample. The SQUID response at this frequency $B_{\mathrm{TF}} \approx \hat{a} \cdot \vec{\nabla}_{r} B_{z}$ where $B_{z}$ is the static magnetic field and $|\hat{a}| \approx 190 \mathrm{~nm}$ is the tuning fork oscillation amplitude. (C) $B_{\text {TF }}$ measured at $B=22 \mathrm{mT}$ after field training to $+200 \mathrm{mT}$ and (D) $-200 \mathrm{mT}$ on the area indicated by the red dashes in inset to (A). The black dashed lines in (C) to $(G)$ indicate the edges of the sample. (E) Half the difference between data shown in (C) and (D). (C) to (E) share the same color scale. Inset: To-scale representations of the SQUID diameter and tuning fork amplitude $\hat{a}$. (F) $B_{z}$ as determined by integrating data in (E). We assume $B_{z}=0$ along the bottom and left edges of the scan range. (G) Magnetization density $m$. Data are presented in units of Bohr magnetons per moiré unit cell area $A \approx 130 \mathrm{~nm}^{2}(4)$. (H) $B_{\text {TF }}$ and (I) $m$ plotted along the line segments indicated in corresponding colors in $(E)$ and $(G)$. The shaded regions in $\mathrm{H}$ show absolute bounds on $m$ obtained by propagating the systematic uncertainty in |â| (24). 

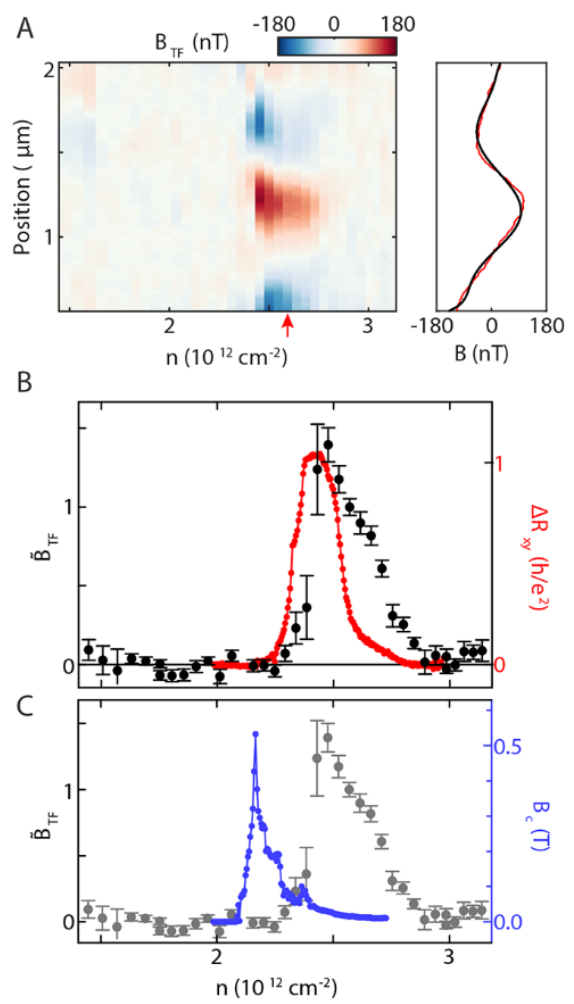

Fig. 2. Density dependence of magnetization. (A) Evolution of $B_{\mathrm{TF}}$ with $n$ in the vicinity of $v=3$, measured along the contour shown in Fig. $1 \mathrm{E}$. The data was taken at $B=44 \mathrm{mT}$ and $T=2.2 \mathrm{~K}$. The trace corresponding to $n$ $=2.57 \times 10^{12} \mathrm{~cm}^{-2}$ is shown in red at right, along with a fit to a 7th order polynomial in black. (B) Comparison of magnetic signal with the residual Hall resistance $\Delta R_{x y}$, shown in red. $B_{\text {TF }}$ traces at different $n$ are fit to the same polynomial as in (A) with a scale factor $\tilde{B}_{\mathrm{TF}}$, which serves as a proxy for $m$. Error bars measure standard error of the mean of the residuals of these fits, normalized to the values shown in the inset of (A). This analysis is covered in more detail in fig. S11 (24). (C) Coercive field $B_{c}$ determined from transport measurements (24) (fig. S8) plotted alongside $\tilde{B}_{\mathrm{TF}}$. These data also appear in the supplementary materials of (6) and are reproduced with permission. 

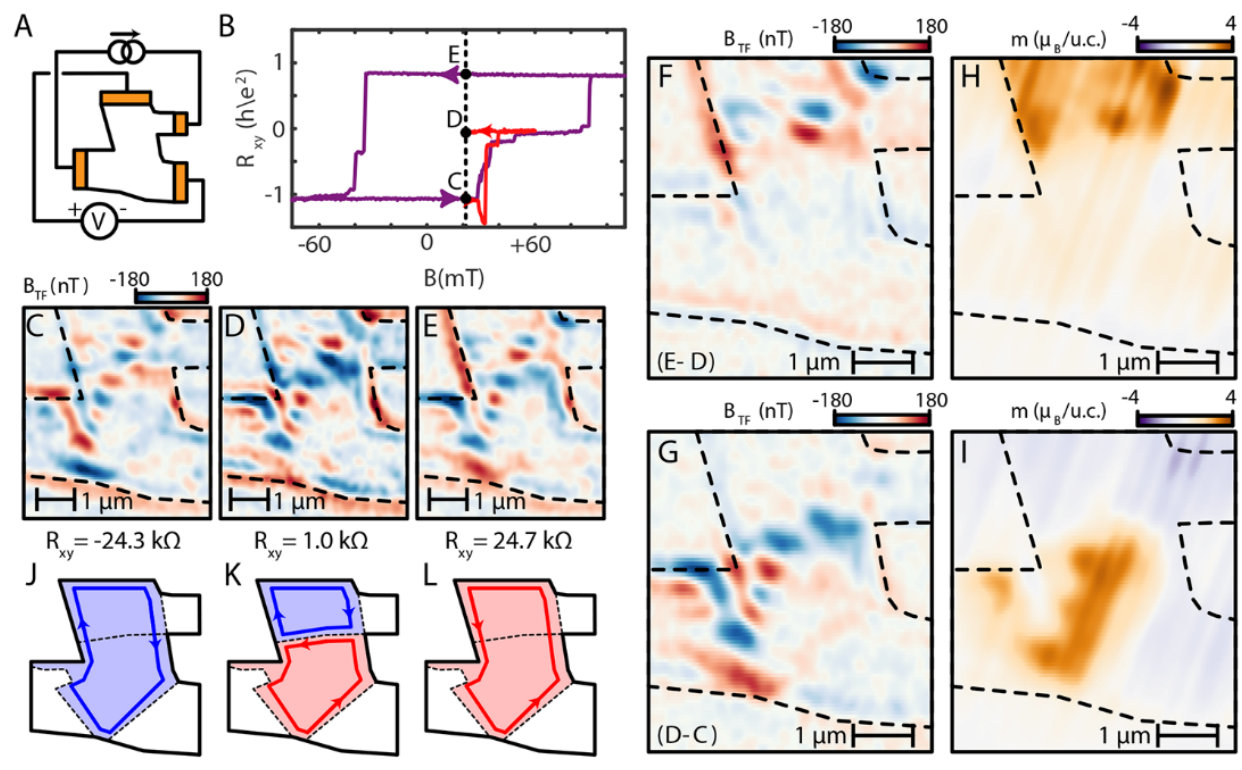

Fig. 3. Mesoscopic magnetic domains. (A) Transport measurement schematic and (B) Hall resistance data as a function of magnetic field for major (purple) and minor (red) hysteresis loops. (C to E) Gradient magnetometry images of the tBLG device at the three indicated values of the Hall resistance. All magnetic imaging presented here was performed at $B=22 \mathrm{mT}, T=2.1 \mathrm{~K}$, and $n=2.36 \times 10^{12} \mathrm{~cm}^{-2}$. ( $\mathrm{F}$ and $\mathrm{G}$ ) Pairwise difference images based on the data presented in $(C)$ to $(E)$. The subtraction is indicated in the lower left corner of each panel. The same domain structures were observed on multiple cooldowns, reminiscent of grains formed by crystalline domain walls in polycrystalline ferromagnetic metals. ( $\mathrm{H}$ and $\mathrm{I}$ ) Extracted magnetization $m$ for the images in $(F)$ and $(G)$. ( $J$ to $L$ ) Schematic depiction of edge state structure corresponding to magnetization states in (C) to (E). Assuming full edge state equilibration, these states would result in $R_{x y}=-h / e^{2}, 0$, and $h / e^{2}$, close to the observed values. 